\title{
Review of "Open Access"
}

Stefanie Haustein ${ }^{1}$

1 University of Ottawa

A general definition of OA by SPARC, an important stakeholder in the scholarly communication community. 